\title{
“Tinder Will Know You Are A 6": Users' Perceptions of Algorithms on Tinder
}

\author{
Christie Abel \\ UC Irvine \\ christie.abel@uci.edu
}

\author{
Lucy Pei \\ UC Irvine \\ lucyp1@uci.edu
}

\author{
Ian R. Larson \\ UC Irvine \\ larsoni@uci.edu
}

\author{
Benedict Salazar Olgado \\ UC Irvine \\ bolgado@uci.edu
}

\author{
Benedict J. Turner \\ UC Irvine \\ benedict.turner@uci.edu
}

\begin{abstract}
Through in-depth interviews of 22 Tinder users, we explore how users interpret their algorithmically mediated experience on the platform. We find that users have various explanations of whether and how Tinder uses algorithms and that users have varying degrees of certainty about these explanations. In response, users report that they act in particular ways given their explanations and degree of certainty. We discuss how users, as part of their sensemaking practice around how algorithms work, engage in forms of improvisation. In addition, we argue that algorithm awareness leads to a more nuanced acknowledgement of inequality and power, including the power-laden roles of platforms themselves.
\end{abstract}

\section{Introduction}

Algorithms are ubiquitously yet quietly embedded into the many interactions people have with, within, and through digital platforms [1, 2, 3, 4]. Algorithm awareness refers to whether users recognize that algorithms mediate and curate their experiences and how they understand these algorithms to work. Prior work has investigated users' awareness of algorithms in platforms like Facebook [5, 6, 7], Google [8], and Twitter [4]. These studies have shown that users are frequently unaware of algorithmic mediation, despite the high degree to which user experiences on those platforms is shaped by the algorithms that underlie those platforms' functions. In cases where users are aware of algorithmic mediation, they typically can only produce partial and unverifiable theories of how the algorithms are mediating their experience $[7,5,6]$.

The increasing role of digital technologies and networked information systems as parts of everyday life has also greatly impacted the world of dating. Notably, mobile dating apps play a growing role in how people meet romantic partners. This growth follows the increased social acceptance of computer-mediated dating as people use apps to meet not only romantic partners but also seek friends and casual encounters [9, 10, 11]. Given the gamut of ways these apps are used, we follow Hutson et al. [12] and refer to them as "intimate platforms." Of these intimate platforms, Tinder is the one of the first dating apps designed specifically for mobile use, and has become one of the most popular [13]. While studies have been conducted on Tinder (e.g. $[14,15])$, it remains a rich space to explore algorithm awareness particularly in relation to intimacy and power. Our study responds to Hutson et al.'s [12] call to interrogate the "design, operation, and role of intimate platforms," by seeking to identify the range of user understandings of how Tinder works.

Through in-depth interviews of 22 Tinder users, we explored how users interpret their experience on the platform and how they act guided by and in response to such interpretations. We focus on user interpretations of the algorithm rather than trying to reverse-engineer the designed unknowable-ness of algorithms belonging to private companies [1]. The users' interpretations of what is shaping their experiences on the platform, accurate or not, are important to study in their own right [5]. We find that users have various explanations of whether and how Tinder uses algorithms and that users have varying degrees of certainty about these explanations. In response, users report that they act in particular ways given their explanations and degree of certainty. We discuss how users engage in forms of improvisation as part of their sensemaking practice around how algorithms work. In addition, we argue that algorithm awareness leads to a more nuanced acknowledgement of inequality and power, including the power-laden roles of platforms themselves.

\section{Related Work}

\subsection{Background and Literature on Tinder}

Entering the market in 2012, Tinder has been an early innovator in the intimate platform space. Tinder utilizes a format that involves swiping on potential 
partners with a double opt-in feature that requires both partners to "like" each other before they can chat. Signing up for a Tinder account is intentionally simple. Profiles consist of one or more photos, along with an open text field in which people can write anything they wish to, from bios to dating preferences. Profiles can also contain links to music streaming services (like Spotify) or social media sites (like Instagram or Facebook). Users then specify gender preferences, age ranges, and a distance radius in miles. Once a user is set up, the main feature of the application is a "deck" of the profiles of potential mates. Each "card" in the deck is the first photo of the potential mate. The user can immediately swipe right on this card to say yes to their half of the double opt-in; immediately swipe left to turn down the potential match; or click to see the written portion of the profile and view other photos. The user can then decide to swipe left or right after viewing the full profile. If both parties swipe right on one another, both receive notifications and a chat room is opened for the two individuals to begin a conversation if they wish to. Additional features have been added over the years to this basic format, including features that are limited behind a paywall [16].

Competing dating apps have refined or added additional features to the Tinder model to give users a more tailored experience. Tinder, in comparison to its competitors, does not clearly document their systems for partner selection; they merely tell their users to "swipe life" as they "Match. Chat. Date" [17]. This purposely opaque design has led many users to construct theories about how the algorithm works. A general survey of internet forums, including Reddit, reveal concerted communal efforts to clarify that which Tinder will not disclose [18]. While the forum-based theories remain speculative, the CEO of Tinder confirmed in 2016 that the platform employs a behind-the-scenes "desirability" ranking algorithm[19]. The designed opacity of the Tinder algorithm leaves room for users to continue to engage in speculation about whether and how their experiences are mediated by algorithms, making Tinder a fruitful site for study.

Previous research on Tinder studied the individual motivators for using the app [20, 21, 22, 23], people's individual behaviors on the app [24, 25], and how both users and Tinder (through use of design features) negotiate authenticity in the space of Tinder [15, 26]. In a survey of dating apps, Tong et. al. found that the design features of dating apps had significant psychological impacts on how users interpreted their feelings of control and satisfaction when ultimately choosing a match [27]. This study taxonomized dating apps into three categories: see-and-screen, in which the user is able to select their favored profiles from a swath of options, algorithm, in which a user is presented with an algorithmically calculated 'best match', and a blended design in which some features from both are employed. However, Tinder's design does not fit in to any of those configurations, catering instead to a new market of users. As described above, Tinder's design focuses on providing users with one profile at a time, but not stating or implying that this one profile has been algorithmically selected to be a 'best' romantic match. Olgado et al.'s [28] study of the ten most popular intimate platforms, which include Tinder, illustrates how users operate within the constraints of a nonneutral infrastructure that mediates user decisions. Research on Tinder's design features has mostly focused on the emotional effect of those features rather than on the ways in which individuals interpret underlying mediating algorithms [14].

\subsection{Algorithm Awareness and Folk Theories}

This paper draws upon the concept of the 'algorithmic imaginary' as developed by Taina Bucher [29]. Bucher argues that while algorithms' exact computational processes are unknown, they "are generative of different experiences, moods and sensations" [29]. Algorithms are capable of affecting and shaping the individual and the social [30, 31]. Despite the fact that algorithms work somewhat behind the scenes, individuals experience their effects in their lives. Drawing upon phenomenology and literature around the concept of affect [32], Bucher argues "we do not necessarily need access to the thing itself (whatever that may be) in order to perceive it." Therefore these perceptions lead to the construction of almost-mythologies by individuals to comprehend the functioning of the phenomenon with which they are faced_- "an algorithmic imaginary."

Similarly to imaginaries, folk theories emerge in scenarios where the operation of a system is opaque to users, leading them to "develop and sometimes share theories about how these curation algorithms work in order to plan their behavior" [5]. Early studies in the field of human-computer interaction focused on designing systems and interfaces so that users could easily develop with a serviceable mental model of how the system was working. The emphasis was for designers and users to operate based on the same mental model. Folk theories, on the other hand, emphasize how the users themselves understand a system to be working, independently of designers. Even when users are presented with expert knowledge of how a system is working, users tend to continue believing their own 
folk theorizations. We subscribe to folk theories in that we value users' explanations in their own right. Whether or not those explanations are accurate to a complex, proprietary, and ever-changing algorithm, the users' beliefs in the explanations they articulate necessarily affects their experience with and through the application.

Acknowledging the impact of algorithms in organizing, selecting, and presenting information on online platforms, previous studies sought to account for how users understand such algorithmic curation. Rader and Gray [33] found that Facebook users have a wide range of differing beliefs and causal inferences in their understanding of how the contents of their News Feeds are filtered by algorithms. These beliefs impact user behavior. A recent study of Airbnb hosts [34] found that understanding of algorithmic curation results in a double negotiation on the platform. Airbnb hosts have to attract not only potential guests but also find means appeal to partially transparent algorithms. These algorithms can also violate identities and needs of users, as in the case of algorithmic exclusion on TikTok, where users develop self-organized practices to resist and circumvent such transgressions [35]. On the other hand, Lee at al. [36] found that drivers of ride sharing services Uber and Lyft have little desire for control over the assignment algorithm. The researchers believe that this might be caused by the drivers' lack of experience and understanding of the system, and so they argue for the importance of supporting social sensemaking around algorithmic systems. DeVito et al. [37] in their study of social media users found that folk theories and understanding of algorithmic mediation of social media feeds are malleable. These may evolve following new information or targeted interventions like sensemaking. These are all in line with Raval et al.'s [38] assertion of the need to investigate how human and social practices relate to algorithmically mediated work and activities.

As these previous studies show, the transparency and operation of algorithms differ between platforms, and they may evolve over time. Further, user understanding of these algorithms vary leading to a wide array of user behaviors and negotiations. Studying Tinder, as an intimate platform with a distinct political economy [28] and as a self-presentation activity [37] expands previous literature while leading to a critical understanding of algorithmically mediated intimacy between users.

\section{Methods}

This research seeks to explore three questions: (1) Do users of Tinder attribute aspects of their user experience to an algorithm? (2) What folk theories, if any, do users develop about how Tinder works? (3) Do users modify their behavior according to perceived algorithms? If so, how?

\subsection{Data Collection and Coding}

This project uses Barney Glaser and Anselm Strauss' [39] grounded theory methodology to inductively understand Tinder users' perceptions of algorithms in the app. GTM offers "the ability to make sense of diverse phenomena, to construct an account of those phenomena that is strongly based in the data ('grounded' in the data), to develop that account through an iterative and principled series of challenges and modifications, and to communicate the end result to others in a way that is convincing and valuable to their own research and understanding" [40]. In applying GTM, we have prioritized reflexivity and openness to the data gathering process, addressing and amending our methodological practices as needed.

This project utilized in-depth interviews (conducted during February 2019) as the key method of data collection. Participants were asked open ended questions about their usage of Tinder, their perceptions of who they interacted with, and their histories using dating apps. Interviews were conducted over the phone or in person and recorded using a dedicated recording device and then manually transcribed. Based on a preliminary sample of interviews, further refinement of the interview questions was undertaken to hone in on emerging trends such as the emergence of profit as a theme. This usage of theoretical sampling was effective for refining our theory, as well as improving subsequent data collection [40]. Data collection was finalized when saturation was achieved. Concurrent with data collection, transcriptions of interviews were independently coded by two members of the research team through the usage of two qualitative data analysis programs Dedoose and NVivo. This usage of constant comparative method between new data and existing codes "combines systematic data collection, coding, and analysis with theoretical sampling in order to generate theory that is integrated, close to the data, and expressed in a form clear enough for further testing" [41]. Lower-level codes applied to individual interviews such as "using it wrong" and "playing Tinder" were combined to produce the axial code "subversion and play" which appears as a theme in our findings.

\subsection{Participants}

The data is the result of in-depth interviews with $22(\mathrm{~N}=22)$ users of Tinder. The initial criteria for participation were being 1) 18 and older and 2) having 
used Tinder within the last 2 years. Based on these criteria, we recruited 22 participants through a mixture of snowball and theoretical sampling. As themes and questions emerged within the data, theoretical sampling was used to target populations most relevant to unanswered and exploratory questions that arose. We ensured diversity in racial and ethnic backgrounds and in sexual orientation in our sampling. Respondents were located in different urban areas in the US, although some had spent time in rural areas as well. All but two of our participants had graduated from or were attending 4-year colleges. In total, our population identified as $64 \%$ female $(36 \%$ Male) with a mean age of 24 (range=18-30). Regarding usage of dating apps outside of Tinder, participants used a wide range of dating apps designed for varying populations and purposes. The dating app Bumble, with its 'female-message-first system', was the most common additional app used by our sample, followed by OkCupid and Hinge. Applications tailored to individuals with specific sexual orientations such as Grindr and Her were also used by several of our respondents.

\section{Results}

We find that participants provided a variety of explanations for how Tinder worked, including explanations that attributed their experience to various types of algorithms. We also found a range of certainty with which participants spoke of their explanations and reasoned about algorithms. Finally, we summarize the range of behaviors that participants reported in response to their understandings of how Tinder works.

\subsection{User Explanations of How Tinder Works}

Many participants shared explanations of how tinder works that attributed curation to an algorithm. Other participants do not implicate an algorithm in their explanation and rather develop their understandings through the other users on the platform. Some of the explanations our participants shared were folk theories, or ideas that are "developed, shared, and circulated by everyday people who are not the expert" [5]. Others were self-generated during the course of our interview, which refers to reasoning that comes from both their own understandings of how they believe the system to work, and their own understandings about how they believe algorithms to work on other systems.

4.1.1. More of the Same/Reinforcement Algorithm One theory advanced by participants attributed curation to a 'reinforcement algorithm.' These participants believed Tinder used an algorithm to curate the people shown based on the user's prior behavior on the app. Participants theorized that Tinder would show more of the types of profiles that one had previously positively interacted with. According to one participant, "I can imagine that depend[ing] on who I swipe, [Tinder] will use that data... to showcase maybe certain types of men more than others" (P3). The reinforcement is perceived as based on identified, marked, and liked similarities that create trends that are further perpetuated. Participant 9 speculated this reinforcement could also be based on similar preferences listed in Tinder: "well you didn't swipe left on a girl that likes country but you did on a girl who likes Indie pop music, so something like that..." This reinforcement algorithm operates as a mechanism of social homophily where contact between similar people is designed to be more likely.

Other users theorized a reinforcement algorithm that had an even broader reach, tracking time spent on each profile. According to Participant 5, "I feel like [Tinder] is trying to match you with certain people. Even though I kept swiping left on people, there were definitely people that I spent longer on their profile, if I knew them or if I thought they were someone attractive. And then they would show up again in the future. I don't know if that's part of the algorithm or just because I had taken longer to look at their profile before so I just recognized them. But I do think that it is trying to find people that you might be interested in and showing them to you." Such a reinforcement system is then based not only on similar categories but also user behavior of time spent.

4.1.2. Compatibility Participants theorized that the profiles that they were being shown were being targeted to them based on the information that they had entered into their own profiles. Participant 9 describes how they believe this to work: “...I would assume [Tinder goes] through everybody's bio and then figure out... On Tinder there's kind of a cap on characters that you can put on your bio. The cap is there for a reason, that way they can actually go in there and scan... up to 300 characters at the output... ". In this case, the participant believes that Tinder is using an algorithm to scan for and categorize similarities in information that was entered on the users' bios and then presenting more compatible profiles as potential matches. Compatibility here then follows and reinforces again the homophilic logic that similarity breeds connection. [42]

4.1.3. Ranking Various users referred to their conception of an algorithm which in some way ranks 
every user according to a numerical scoring system. Many participants believed that this score was generated based on their own swiping habits, as well as the swiping habits of others. According to Participant 1, "It kinda feels like Tinder knows... You start thinking in numbers. Tinder will know you are six. [...] you will see someone and you will know if they have swiped on you... and Tinder thinks you are somewhat compatible in terms of [...] attractiveness...”.

Others theorized that this numeric score is co-determined by both Tinder's conception of a users' 'attractiveness' as well as the behaviors of other users. Participant 17 explains, "I think...you probably get pushed to the top of stacks if you are an attractive profile. Like if you get a lot of rights. There's probably some benefits to be more attractive."

4.1.4. Profit Participants theorized that Tinder was showing them profiles using an algorithm that incorporated payment structures. Participants believed that users who paid Tinder for premium features (such as Tinder Gold or Tinder Plus) would be favored by the algorithm. According to Participant 4, "I assume that people who pay for it appear more on people's, like appear in more people's decks, maybe people who get more matches also appear in more people's decks, or maybe people who get less, like people who are new, like appear more, just so they can get immersed in it more, like I don't know, like there's a handicap for newbies, effectively."

Other users theorized that their presence on the app was a way for Tinder to extract money, so Tinder's algorithm would work to keep them active. According to Participant 18, "Tinder might have created some profiles just to keep their male users active. Seems like some of them weren't even trying to get anything, just profiles that were there." Participant 18 went on to note, "My guess would be that it's targeted toward serving its paying customers more. So I'm probably more so seeing profiles of people who are paying to use the app. Oh yeah, I know [Tinder has] that search feature for people who pay. So I'm sure I'm seeing people who are paying to be searched. And I feel like I might be seeing people who swiped on me who don't have a lot of matches. If they're trying to not make people feel like it's not worth it to use the app." Users acknowledge the profit driven bait-and-switch quality of how the app works.

In line with the theory that Tinder's algorithm was designed to keep people on the app, Participant 2 states, "[Tinder's] goal is not show me just people I will actually like or connect with. Their goal is to make money off of me and keep me here, not with somebody.
That's the clincher... It becomes very clear that maybe someone else is out there and we're participating in this terrible system and maybe we could miraculously meet, but we are using the thing that is also using us." Here we can see how participants both acknowledge and seemingly surrender to this profit motive which they are critical of.

\subsubsection{Non-speculative Explanations Some} individuals drew on the documentation and interface of Tinder to argue the profiles they see were chosen due to their personal choices over age, sex, and location. This, by definition, points towards a belief in algorithmic sorting, albeit one that is simple and only draws upon these three variables. Participants who did not theorize a more complex algorithm believed that beyond these overt variables, the curation occurred randomly. According to Participant 4, "I think Tinder thinks less, like I really don't think there's much behind it. It really seems very random and just kind of showing you just a randomized version of everyone who's within your area. Or at least I feel like the other apps will have intro pages, that are, oh, like we think really hard to like put this deck together for you, but Tinder is just like no, your deck is just there, you know, you just swipe, like there's no kind of like guided tour of like, oh we're thinking behind the scenes."

We encountered both participants who brought up the term "algorithm" on their own and those who did not mention the term until we asked directly about algorithms. In the next section, we will discuss the degrees of certainty and ways in which participants talked about their explanations of how Tinder works.

\subsection{Degrees of (Un)Certainty in User Explanations}

Throughout our interviews, some participants expressed a very clear and well researched knowledge of the Tinder algorithm, whilst others were more tentative. This section will explore the different degrees of certainty and doubt with which participants shared their explanations of how Tinder works.

4.2.1. Acquired Knowledge Some individual expressed clearly-formulated ideas of the inner workings of Tinder. These participants reported finding out from some external source how Tinder worked. Participant 11 explains their understanding: "The Tinder Elo? Oh yeah, I heard about it from a friend. It's like if you find, if a desirable person swipes right on you, and then your rating goes up, but if you continue 
to swipe right on people deemed by the algorithm as undesirable it starts to lower its expectations of you, and I've like known a few people who've tried to figure it out and like optimize their swiping to see quote unquote better people, but that's far more hardcore than I am. A lot of it are like the programmer types trying to beat the system."

The example above speaks to how some participants get their knowledge of the algorithm from peers. By talking and sharing about perceptions of how algorithms work, users engage in algorithmic gossip [43] that consequently shape their responses and practices. Algorithmic gossiping can also be influenced by other external sources. Participant 14 notes, "I briefly once started an article, but I didn't finish it, on how the algorithm did apparently rank people in terms of their attractiveness score, attractiveness meaning not necessarily just what you look like, but also how many people on the app are swiping right on you, or [...] desirableness."

These explanations which carry a high degree of certainty and clarity are akin to folk theories, which are explanations that have gained traction in certain communities about how certain systems work [5] As explanations such as desirability rankings have circulated online, and reached participants through external sources, participants are able to provide a read-made and succinct answer about how algorithms are mediating on Tinder.

4.2.2. Algorithmic Improvisation In comparison to the theme of knowing, with its solid conclusions and clear articulations, this theme draws out the more tentative moments of algorithmic sensemaking. Some participants have a tenuous understanding of algorithms in Tinder, which are contextualized by their broader experiences in their socio-technical lives. Participants who did not directly bring up an algorithm would begin to speculate about the algorithm when directly asked about it. They would transfer their understanding of algorithms in other digital platforms to begin to guess at how an algorithm might be working in Tinder.

In this way, individuals demonstrated that they were capable of improving an understanding on the spot about the functioning of the algorithm. When we asked follow-up questions after a user mentioned an algorithm in passing, individuals often initially expressed doubt or emphasized that they did not know how the algorithm worked. However, following this, individuals would reason aloud and slowly build their understanding of the algorithm. In contrast with the clearly structured answers from prior research, these participants would take time, and often loop back on themselves to clarify and develop points. This theme is further elaborated in the discussion section.

4.2.3. "I Don't Know" Our interviews revealed moments wherein users believed in the existence of the algorithm, but could not express how it worked or expressed doubt in their interpretation. Rather than responding with a committal "no" to a direct question of whether algorithms were present on the app, participants who expressed the least algorithmic awareness typically responded with "I don't know" to any questions about algorithmic mediation.

"I don't know" was also often used by participants to express doubt or uncertainty, tagged onto a longer exposition about the existence of the algorithm or about its functioning. Participant 5 notes, "I don't know if that's part of the algorithm or just because like I had taken longer to look at their profile before..." (P5). This participant expressed uncertainty between two possible explanations, involving different degrees of algorithmic responsiveness to their actions. The participant conceives of both a more hard-coded algorithm and one that considers the amount of time the user spends looking at a profile. Another participant tagged on "I don't know!" after coming upon a possible contradiction in their line of reasoning: "If tinder's algorithm is only based on who likes me, then I constantly would have to change so that more people who like me. I don't know!" (P2).

\subsection{Behavioral Responses to Understandings of Tinder}

After asking respondents about how they thought Tinder worked, we used exploratory probing questions to evaluate whether or not they responded to the perceived workings of Tinder in any way. We found that some users sought to improve their outcomes for the intended purpose of finding potential dates or other intimate relationships, while others acknowledged that they could change their behavior based on their understandings but chose not to. Other users reported subversive and playful behaviors based on their understanding of how Tinder works.

4.3.1. Trying to improve matching Based on their understanding of how Tinder works, users reported following what they saw as popular or helpful in achieving the goal of meeting potential matches. With the understanding that profile setup mattered, a participant reported, "Yeah, I tried to set it up so I 
would find like-minded people. I tried flattering pics and writing a short and interesting bio" (P22). Another user reported changing his profile based on feedback and trying to mirror what he was looking for: "Based on feedback from my last partner I put that I'm a vegetarian in my profile. That I'm bi...I need to see that you have a life, not necessarily like friends, but stuff that you do outside of your own house. So I try to show that. So like outdoorsy stuff, pictures with my friends in them. Those are the things that I look for in people's profiles. So I kind of try to mirror that in my profile" (P21). Improving profiles can then be driven by the felt need to respond to feedback or to position oneself more strategically.

4.3.2. Opting out of Optimizing In contrast to users who changed their behaviors to improve outcomes on Tinder based on their perception of algorithms and people's preferences, some respondents reported ignoring this knowledge. Users like Participant 11 acknowledge that an algorithm exists and attribute certain factors to it, but actively stated that they were not compelled to change their habits to "optimize" to the perceived algorithm. "I've heard of like that Tinder Elo algorithm but I've never really, I don't know, I know a few people who have like gone all in on like optimizing theirs to see like hot people, but I don't, I haven't gotten into that".

Paralleling the algorithm-perceiving participants, respondents who attributed patterns to non-algorithmic causes would choose to not change their habits in response to trends they were seeing. Having previously touched upon patterns seen in the profile of potential matches, Respondent 9 had this to say about their own habits: "I was talking to a friend who... showed me his profile and in the same way that the girls were doing it with travel, he had the same kind of things, but a male version of it. Like, [including] pictures of him at his office. I didn't say anything or ask him to tell me why he did, but I found it interesting that he is also doing the same thing. I didn't have any of that. I'm more so just trying to be goofy and I think because of that I didn't get a lot of hits. I didn't want to succumb myself to being that basic" (P9). This active refusal to change despite knowing trends that led to more matches on the platform was echoed in several participants.

4.3.3. Subversion and Play Participants reported subversion and play with the premise of Tinder as an intimate platform. These subversive behaviors happened in response to both algorithmic and non-algorithmic understandings of how Tinder worked.
One participant, who shared a theory of a reinforcement algorithm as driving Tinder's functions, shared: "I don't know if Tinder has some sort of algorithm where they try to pick out similar profiles. I just assumed that social media in general does that. But I didn't want that to happen with Tinder necessarily. So that's why I just kept swiping left. I didn't really want to engage with anyone on there either." (P5). This participant goes on to note that, having perceived this habit-learning algorithm, she sought to deprive the app of valuable information that could be used to calculate or learn their patterns. "Because I didn't swipe right so I feel like it was hard for Tinder to really get any preferences or try to get any trends out of it." (P5). By willfully denying the platform the data that she perceived to be feeding the algorithm at work, this user attempted to subvert the power of Tinder to know her.

With an understanding of Tinder as being mostly driven by user preferences, some participants attempted to subvert these trends. Participant 3, who reflected considerably on being a racial and cultural minority on Tinder, noted the following: "As a form of resistance, because on some of these apps you see a lot of asshole level descriptors, like masc for masc. No fem. No Asians. So I wrote; Asian for Asian. POC for POC. Educated 4 Educated. As a way to mock the assholes" (P4). Additionally, they commented on playfully resisting general trends: "People on tinder tend to use...the personality, INFJ, personality types. Myer Briggs. I absolutely despise [them]. So I mock them. When you open my profile it'll say GPKJ. Those are just my initials with a random J added. I think it's funny.”(P4).

A participant who was a self-proclaimed catfish, or a person who misrepresents themselves on social media platforms for the purpose of luring or playing with an unknowing user, used their perception of trends to model behaviors that would lead to more fruitful exploitation of the app. "I was kind of ironic with my other profile too, so I would ask my friends, do you think this is hot? And depending on if they said yes I would use it. Like do you think I could catch a sad straight white guy with this? And they're like oh yeah, so I'd use it. That's all the thought I put into it. I also put a picture of me [in a green bodysuit] which was like a wild card, like it was a weird picture" (P4). Although their actions align with those who attempted to improve their results on Tinder, her purpose was subversive in "catching" other users to prank them.

Playing with non-algorithmic patterns was common among participants. Participant 9 described a social game created around the process of swiping: "I've heard of Tinder drinking games that people have together. 
One of my friends was telling me that a girl that he met would play Tinder roulette; if you see a shirtless guy. How many shirtless pictures you would see equals how many shots you would take" (P9). These sorts of playful applications acknowledge the dominant trends of shirtless men or images commonly seen as attractive while asserting agency in engaging with them differently, through subversion and play.

\section{Discussion}

\subsection{Improvisation and Sensemaking}

We found that, similar to prior research on algorithm awareness $[6,5,7,29]$, when participants had no prior ideas about how Tinder's algorithm might work, they employed abductive reasoning to develop their theories for how they believed the system to work, or how they believed that certain things were happening in-app. Particularly, they used their own observations of what they were shown on the app to make sense of how they believed the algorithm to work.

One of the key findings from this paper is the demonstration that individuals deploy their understandings of what an algorithm is in an improvisatory manner. Often, interviewers got to witness an individual verbally working through how they thought the Tinder algorithm works. Following the direct question near the end of the session or following a follow up question about algorithms after it was mentioned in passing, users would express that they had no prior knowledge of the algorithm. However, many would then continue, and begin to lay out how they think the algorithm works by drawing upon their technical knowledge (if any) and the experiences of the app. This is a valuable funding as it shows how this individual conceptualized the capabilities of algorithmic reasoning. What do individuals think a computable phenomenon is? What is data for an algorithmic system? Such questions hovered below the surface as participants considered the possibility and implications of algorithmic mediation in Tinder.

This heavily reflects the broader techno-social landscape of the user, and what they understand computation and the abstract "algorithm" as capable of doing. It shows that the very basis for a belief in an algorithmic process can come from their own theoretical understanding of algorithms. In contrast to participants who held a solid belief, often constituted by prior research, and outlined their theory clearly to the interviewer in absolute terms, participants who openly reasoned were more tentative, often going back and correcting themselves. This relates to prior findings where folk theories about social media feeds were found to fall into categories of "malleable" and "rigid" [37]. Our participants' explanations reflected a process of improvising on the spot, while DeVito et al. probed at the malleability of folk theories. In many respects, just as we can ask 'what rhythm and chord sequences are limiting and defining a music improvisation?', we can ask 'what are the limits to this algorithmic improvisation?'. The focus here is not on participants' hurried conclusion, but instead is their practice of algorithmic sensemaking.

\subsection{Making Sense of Algorithms and Power}

Our findings on behavioral modifications reveal a more complex procedure of negotiation between perceived patterns. Instead of amending habits to fit with the perceived algorithm or trends other users were exhibiting, many of our respondents shared ways in which they subvert and resist. Whereas previously literature exemplifies the fact that computational systems and algorithms have problematic realities for individuals who do not fit into the socially constructed image of beauty, our findings shed light on how users are non-neutral recipients of potential oppression. Simple interactions and presentations can be used as opportunities for resistance or distorting the algorithm. Social activities and trickery can be users' responses to standardized trends being observed. Corresponding with previous literature, users do optimize and attempt to control their presentation in pseudo-mathematical terms [44]. Respondents sought to deny the app information and use the app for drinking games and pranks beyond the intended usage as a platform for intimate relationships.

As Virginia Eubanks discusses, computing arrangements are rooted in histories of privilege and power [3]. This power has been transferred into the machine, reproducing the same systems of inequality while remaining invisible to the unaware eye. Search engines such as Google have already been shown to promote a standardized image of beauty that is most closely associated with whiteness, while simultaneously more often linking blackness with explicit and dehumanizing images [45]. It is perhaps no secret that dating platforms like Tinder collect data from users to better predict user habits. Former OkCupid CEO Christian Rudder [46] acknowledges that platforms like OkCupid and Tinder reproduced racial hierarchies where in certain minority groups receive less attention and play from the user base.

Homophily has been found to be prevalent on dating platforms like Tinder [47], as well as homogeneity in 
cultural markers affiliated with upper class pursuits (eg. traveling, hiking, etc). Ways of presenting on Tinder are standardized and constructed through a casting mold, [28] one that is closely linked to socio-economic status, gender conformity, and race. This homogeneity becomes more problematic when racialized practices become subsumed into everyday practices. This racial effect, tied with practices that reproduce and affirm cultural hierarchies, have the potential to create an algorithmically marginalized group on platforms like Tinder.

Critically accounting for and engaging with algorithms as sociotechnical systems, Bucher [29] argues for a theoretical framing of and methodological approaches to understanding how algorithms in their ontological multiplicity are reflective, constitutive, and generative of power and politics in relation to social agencies and practices. The variable user ontology and epistemology of algorithms which we have laid out are "never reducible to questions of materiality or perception" [29]. Our engagements with participants regarding the Tinder algorithm showed that some users take an improvisatory stance toward the possibility of algorithmic mediation. Between unawareness and learned folk theories lies an area where users may speculate and then retract and build upon their earlier speculations about how the algorithm may be mediating their experience. This open-ended thinking about the potentials of algorithmic mediation led some participants to look more at the structural dynamics of the platform. It was after several iterations of reflection on how Tinder works that P2 made a critique of the platform's profit structure, noting that the system seeks to keep users swiping as paying customers rather than facilitating meaningful connections that would lead people to leave the app after finding a relationship.

Research has established a problematic lack of algorithm awareness among users, which we also find to an extent. HCI researchers have agreed that "folk theories" even if incorrect largely shape user experiences with technology. We add to this that users can improvise, sometimes in critical directions, about the role of algorithmic mediation in their experiences. This flexible act of reflection important to study in its own right alongside the different ways in which folk theories diffuse.

\section{Conclusion}

This study has shown that users of the dating platform Tinder have varying degrees of algorithm awareness, and that they respond according to their perceptions. As an emerging emphasis in $\mathrm{HCI}$, we believe that this study has practical implications for future research on the human-algorithm experience. This study was limited by the small sample size and specificity of the Tinder platform. Future research could explore additional intimate platforms (Bumble, Hinge, OkCupid, etc.) and expand the scope of the sample to include populations underrepresented in our data. Incorporating expert testimony from developers could also serve as a counterpoint to the perceived theories of general users. Additionally, future research can be undertaken on themes and trends that inductively emerged within the data, particularly on gamification of dating applications and the digital labor of using apps like Tinder. We believe by expanding on these areas of inquiry that we can better situate Tinder within a larger socio-cultural discussion of the increasing role of digital technologies and networked information systems as parts of everyday life.

\section{References}

[1] J. Burrell, "How the machine 'thinks': Understanding opacity in machine learning algorithms," Big Data \& Society, vol. 3, no. 1, p. $2053951715622512,2016$.

[2] L. D. Introna and H. Nissenbaum, "Shaping the web: Why the politics of search engines matters," The information society, vol. 16, no. 3, pp. 169-185, 2000.

[3] V. Eubanks, Automating inequality: How high-tech tools profile, police, and punish the poor. St. Martin's Press, 2018.

[4] J. Kulshrestha, M. Eslami, J. Messias, M. B. Zafar, S. Ghosh, K. P. Gummadi, and K. Karahalios, "Quantifying search bias: Investigating sources of bias for political searches in social media," in Proceedings of the 2017 ACM Conference on Computer Supported Cooperative Work and Social Computing, pp. 417-432, ACM, 2017.

[5] M. Eslami, K. Karahalios, C. Sandvig, K. Vaccaro, A. Rickman, K. Hamilton, and A. Kirlik, "First i like it, then i hide it: Folk theories of social feeds," in Proceedings of the 2016 cHI conference on human factors in computing systems, pp. 2371-2382, ACM, 2016.

[6] M. Eslami, A. Rickman, K. Vaccaro, A. Aleyasen, A. Vuong, K. Karahalios, K. Hamilton, and C. Sandvig, "I always assumed that i wasn't really that close to [her]: Reasoning about invisible algorithms in news feeds," in Proceedings of the 33rd annual ACM conference on human factors in computing systems, pp. 153-162, ACM, 2015.

[7] T. Bucher, "Want to be on the top? algorithmic power and the threat of invisibility on facebook," New media \& society, vol. 14, no. 7, pp. 1164-1180, 2012.

[8] T. Gillespie, "The relevance of algorithms," Media technologies: Essays on communication, materiality, and society, vol. 167, 2014.

[9] R. N. Crooks, "The rainbow flag and the green carnation: Grindr in the gay village," First Monday, vol. 18, no. 11, 2013. 
[10] M. J. Rosenfeld and R. J. Thomas, "Searching for a mate: The rise of the internet as a social intermediary," American Sociological Review, vol. 77, no. 4, pp. 523-547, 2012.

[11] D. Zytko, S. A. Grandhi, and Q. Jones, "Frustrations with pursuing casual encounters through online dating," in Proceedings of the 33rd annual ACM conference extended abstracts on human factors in computing systems, pp. 1935-1940, 2015.

[12] J. A. Hutson, J. G. Taft, S. Barocas, and K. Levy, "Debiasing desire: Addressing bias \& discrimination on intimate platforms," Proceedings of the ACM on Human-Computer Interaction, vol. 2, no. CSCW, pp. 1-18, 2018.

[13] K. Jackson, “A brief history of online dating," 2018.

[14] G. David and C. Cambre, "Screened intimacies: Tinder and the swipe logic," Social media+ society, vol. 2, no. 2, p. $2056305116641976,2016$.

[15] S. Duguay, "Dressing up tinderella: Interrogating authenticity claims on the mobile dating app tinder," Information, Communication \& Society, vol. 20, no. 3, pp. 351-367, 2017.

[16] T. H. Desk, "A guide to tinder."

[17] Tinder, "Tinder official website."

[18] Reddit, "Tinder subreddit."

[19] A. Carr, "What's really going on inside tinder?," 2016.

[20] E. Timmermans and E. De Caluwé, "To tinder or not to tinder, that's the question: An individual differences perspective to tinder use and motives," Personality and Individual Differences, vol. 110, pp. 74-79, 2017.

[21] G. Ranzini and C. Lutz, "Love at first swipe? explaining tinder self-presentation and motives," Mobile Media \& Communication, vol. 5, no. 1, pp. 80-101, 2017.

[22] E. Timmermans, E. De Caluwé, and C. Alexopoulos, "Why are you cheating on tinder? exploring users' motives and (dark) personality traits," Computers in Human Behavior, vol. 89, pp. 129-139, 2018.

[23] S. R. Sumter, L. Vandenbosch, and L. Ligtenberg, "Love me tinder: Untangling emerging adults' motivations for using the dating application tinder," Telematics and Informatics, vol. 34, no. 1, pp. 67-78, 2017.

[24] L. E. LeFebvre, "Swiping me off my feet: Explicating relationship initiation on tinder," Journal of Social and Personal Relationships, vol. 35, no. 9, pp. 1205-1229, 2018.

[25] C. L. Mason, "Tinder and humanitarian hook-ups: The erotics of social media racism," Feminist Media Studies, vol. 16, no. 5, pp. 822-837, 2016.

[26] R. R. Silva, M.-L. Koch, K. Rickers, G. Kreuzer, and S. Topolinski, "The tinder ${ }^{\mathrm{TM}}$ stamp: Perceived trustworthiness of online daters and its persistence in neutral contexts," Computers in Human Behavior, vol. 94, pp. 45-55, 2019.

[27] S. T. Tong, J. T. Hancock, and R. B. Slatcher, "Online dating system design and relational decision making: Choice, algorithms, and control," Personal Relationships, vol. 23, no. 4, pp. 645-662, 2016.

[28] B. S. Olgado, L. Pei, and R. Crooks, "Determining the extractive casting mold of intimate platforms through document theory," in Proceedings of the 2020 CHI Conference on Human Factors in Computing Systems, pp. 1-10, 2020.
[29] T. Bucher, "The algorithmic imaginary: exploring the ordinary affects of facebook algorithms," Information, Communication \& Society, vol. 20, no. 1, pp. 30-44, 2017.

[30] D. Beer, Popular culture and new media: The politics of circulation. Springer, 2013.

[31] R. Kitchin and M. Dodge, Code/space: Software and everyday life. Mit Press, 2011.

[32] Z. Papacharissi, Affective publics: Sentiment, technology, and politics. Oxford University Press, 2015.

[33] E. Rader and R. Gray, "Understanding user beliefs about algorithmic curation in the facebook news feed," in Proceedings of the 33rd annual ACM conference on human factors in computing systems, pp. 173-182, 2015.

[34] S. Jhaver, Y. Karpfen, and J. Antin, "Algorithmic anxiety and coping strategies of airbnb hosts," in Proceedings of the 2018 CHI Conference on Human Factors in Computing Systems, pp. 1-12, 2018.

[35] E. Simpson and B. Semaan, "For you, or for" you"? everyday lgbtq+ encounters with tiktok," Proceedings of the ACM on Human-Computer Interaction, vol. 4, no. CSCW3, pp. 1-34, 2021.

[36] M. K. Lee, D. Kusbit, E. Metsky, and L. Dabbish, "Working with machines: The impact of algorithmic and data-driven management on human workers," in Proceedings of the 33rd annual ACM conference on human factors in computing systems, pp. 1603-1612, 2015.

[37] M. A. DeVito, J. Birnholtz, J. T. Hancock, M. French, and S. Liu, "How people form folk theories of social media feeds and what it means for how we study self-presentation," in Proceedings of the 2018 CHI conference on human factors in computing systems, pp. 1-12, 2018.

[38] N. Raval and P. Dourish, "Standing out from the crowd: Emotional labor, body labor, and temporal labor in ridesharing," in Proceedings of the 19th ACM Conference on Computer-Supported Cooperative Work \& Social Computing, pp. 97-107, 2016.

[39] B. G. Glaser and A. L. Strauss, Discovery of grounded theory: Strategies for qualitative research. Routledge, 2017.

[40] M. Muller, "Curiosity, creativity, and surprise as analytic tools: Grounded theory method," in Ways of Knowing in HCI, pp. 25-48, Springer, 2014.

[41] C. Conrad, Qualitative research in higher education: Experiencing alternative perspectives and approaches. Ginn Press, 1993.

[42] W. H. K. Chun, "Queerying homophily," 2018.

[43] S. Bishop, "Managing visibility on youtube through algorithmic gossip," New media \& society, vol. 21, no. 11-12, pp. 2589-2606, 2019.

[44] M. Condis, Gaming Masculinity: Trolls, Fake Geeks, and the Gendered Battle for Online Culture. University of Iowa Press, 2018.

[45] S. U. Noble, Algorithms of oppression: How search engines reinforce racism. NYU Press, 2018.

[46] OkCupid, "Race and attraction, 2009-2014," 2014.

[47] K.-H. Lin and J. Lundquist, "Mate selection in cyberspace: The intersection of race, gender, and education," American Journal of Sociology, vol. 119, no. 1, pp. 183-215, 2013. 